\title{
Expression of Bcl-2 is a favorable prognostic biomarker in lung squamous cell carcinoma
}

\author{
CHANGJIANG FENG $^{1 *}$, JIAQI WU ${ }^{2 *}$, FAN YANG $^{1}$, MANGTANG QIU $^{1}$, SHUOFENG HU $^{2}$, \\ SAISAI GUO ${ }^{3}$, JIN WU ${ }^{3}$, XIAOMIN YING ${ }^{2}$ and JUN WANG ${ }^{1}$ \\ ${ }^{1}$ Department of Thoracic Surgery, Peking University People's Hospital, Beijing 100044; \\ ${ }^{2}$ Computational Omics Laboratory, Beijing Institute of Basic Medical Sciences; ${ }^{3}$ State Key Laboratory of Proteomics, \\ Institute of Basic Medical Sciences, National Center of Biomedical Analysis, Beijing 100850, P.R. China
}

Received February 7, 2017; Accepted January 22, 2018

DOI: $10.3892 / \mathrm{ol} .2018 .8198$

\begin{abstract}
Lung squamous cell carcinoma(LUSC) is the second major type of lung cancer globally. The majority of patients with LUSC are clinically diagnosed at the advanced stages, thus it is urgent to identify suitable prognostic markers for LUSC. B-cell lymphoma 2 (Bcl-2) has been widely studied in non-small cell lung cancer (NSCLC). However, the prognostic role of Bcl-2 in NSCLC remains conflicting and controversial, particularly for LUSC. Although certain studies have been performed to identify the prognostic value of $\mathrm{Bcl}-2$, to the best of our knowledge, no study has investigated the prognostic role of Bcl-2 in LUSC specifically. The present study aimed to comprehensively evaluate the prognostic value of $\mathrm{Bcl}-2$ in LUSC. Microarray data for LUSC were downloaded from public databases, including the Gene Expression Omnibus and The Cancer Genome Atlas. Microarray data of 901 patients with LUSC from 16 data sets were retrieved. The meta-z algorithm was applied and the combined $\mathrm{z}$ score was identified as -2.43 , suggesting Bcl-2 is a favorable prognostic biomarker. Furthermore, immunohistochemical staining of Bcl-2 expression was performed in a tissue microarray of 72 patients with LUSC and survival analysis demonstrated that patients with high expression Bcl-2 exhibited significantly more improved overall survival rates compared with those with low Bcl-2 expression. Multivariate Cox regression revealed that high
\end{abstract}

Correspondence to: Professor Jun Wang, Department of Thoracic Surgery, Peking University People's Hospital, 11 Xizhimen South Street, Xicheng, Beijing 100044, P.R. China

E-mail:wangjun@pkuph.edu.cn

Professor Xiaomin Ying, Computational Omics Laboratory, Beijing Institute of Basic Medical Sciences, National Center of Biomedical Analysis, 27 Taiping Road, Haidian, Beijing 100850, P.R. China E-mail: yingxm@bmi.ac.cn

*Contributed equally

Key words: B-cell lymphoma 2, lung squamous cell carcinoma, prognosis, biomarker expression of $\mathrm{Bcl}-2$ is an independent favorable prognostic factor (hazard ratio, 0.295; confidence interval, 0.097-0.904; $\mathrm{P}<0.05)$. Therefore, the results of the present study demonstrated that $\mathrm{Bcl}-2$ is a favorable prognostic biomarker in LUSC.

\section{Introduction}

Lung cancer is currently the leading cause of cancer-associated mortality worldwide (1). The majority of lung cancer cases are non-small cell lung cancer (NSCLC), which accounts for $\sim 85 \%$ of newly diagnosed lung cancer cases (2). Lung adenocarcinoma (LUAD) and lung squamous cell carcinoma (LUSC) are the two most common histologic subtypes of NSCLC (3).

In recent years, with the progression of molecular medicine and the emergence of targeted drugs, treatment of NSCLC started to become individualized molecular targeted 'precise' treatment (4). At present, the individualized molecular targeted therapy for clinical application is primarily for epidermal growth factor mutation and anaplastic lymphoma kinase fusion genotypes in lung cancer, both of which have clear molecular targets, and targeted drug has significantly improved the clinical efficacy $(5,6)$. However, the prognosis of LUSC remains poor and identifying effective prognostic biomarkers for LUSC remains urgent (7-9).

B-cell lymphoma 2 ( $\mathrm{Bcl}-2)$ is an antiapoptotic protein, which belongs to the Bcl-2 family. It is located in the inner mitochondrial membrane and to a lesser extent in cell membranes (10). The primary function of Bcl-2 appears to be to inhibit apoptosis (programmed cell death) and to prolong cell survival by arresting cells in the G0/G1 phase of the cell cycle $(11,12)$. Previous studies have revealed that $\mathrm{Bcl}-2$ is highly expressed in several hematologic and solid malignancies, including acute lymphocytic leukemia (ALL), breast, prostate, colorectal, lung, stomach, and ovarian cancer (13-15).

It has been confirmed Bcl-2 as an independent prognostic marker in breast cancer (16), and Bcl-2 is also considered to be a favorable prognostic marker in NSCLC (17-19), but Kim et al (20) reported that Bcl-2 is an adverse prognostic marker. The aforementioned studies focused on NSCLC or LUAD; however, to the best of our knowledge, there is no previous study regarding the role of Bcl-2 in LUSC (21). LUSC and LUAD are very different in the molecular biological 
background and clinical biological characteristics: LUAD has more driver genes, including EGFR and ALK, which also means that there are more clinical treatment options, and LUAD is more common in non-smokers, compared with LUSC $(9,22,23)$.

Over the past decade, high-throughput detection technology has yielded a mass of tumor data (24), but these datasets are scattered, due to patient cohort, technology platform and other heterogeneous variables, thus making it hard to compare (22). In the present study, in order to solve this problem and to make better use of these public database (25), 901 LUSC gene expression profiles were integrated and the association between expression level and overall survival was analyzed. Furthermore, the prognostic value of Bcl-2 in LUSC was validated with a tissue microarray (TMA) using immunohistochemistry (IHC) analysis.

\section{Materials and methods}

Data collection and prognostic association meta-analysis. Data from 16 publicly available microarray studies on lung cancer with overall survival outcome data from the National Center for Biotechnology Information Gene Expression Omnibus (25) and The Cancer Genome Atlas were curated. Raw CEL files were obtained where possible, and were normalized, summarized and log-transformed using robust multi-array average function of the affy $\mathrm{R}$ package (https://www.r-project.org/; R version 3.1.2; affy version 1.44). The probe-based expression was converted into gene expression profiles, and only cases on patients with squamous cell carcinoma were retained for subsequent analysis.

For the meta-analysis of the prognostic association between gene expression and survival outcomes, the statistical significance was assessed by z-scores via univariate Cox proportional hazards regression in each of the 16 datasets (Table I) using the coxph function of the survival $\mathrm{R}$ package (26). Z-scores represent the number of standard deviation below (or above) the mean of a normal distribution. In addition, $\mathrm{z}$-scores conveniently reflect the directionality and significance of statistical association. In order to obtain the integrated and robust prognostic landscape, $\mathrm{z}$-scores for a gene were summarized across all 16 datasets to yield a 'meta-z-score' for the prognostic significance assessment using Lipták's weighted meta-z test with weights set to the square roots of sample sizes. The prognostic genes were defined by meta-z-scores filtered for Imeta-z| $>1.96$ ( $|z|>1.96$ is equivalent to a two-tailed $\mathrm{P}<0.05)$. Favorable prognostic genes have meta-z $<-1.96$ and adversely prognostic genes have meta- $\mathrm{z}>1.96$.

Patient tissue samples. A total of 72 patients LUSC who were diagnosed, and underwent surgery in People's Hospital of Peking University (Beijing, China) between 2004 and 2010 were enrolled in the present study. Fresh LUSC tissue from each patient were formalin-fixed, paraffin-embedded (27), and constructed into TMAs (Shanghai Outdo Biotech Co., Ltd, Shanghai, China): Using the tissue chip spotter, the marked tissue is arranged on the blank wax block according to the design, and then the slicing machine is used to slice the array wax block continuously to obtain the tissue chip. Postoperative follow-up has lasted $\geq 3$ years for all patients. Histopathological evaluation was performed independently by two pathologists. The clinical stage of the tumors was evaluated by an experienced pathologist according to the 7th edition of the American Joint Committee on Cancer (AJCC) tumor node metastasis (TNM) staging system (28). Complete clinical information, including age, gender, stage, smoking, follow-up time, and survival status was collected. The present study was approved by the Institutional Review Board of the People's Hospital of Peking University and written informed consent was obtained from each patient.

IHC analysis. All hematoxylin and eosin slides were centrally reviewed at the Department of Pathology in People's Hospital of Peking University according to the histopathological classification system adopted by the World Health Organization to confirm tumor type (29). TMA block sections (4- $\mu$ m thick) were rewarmed in the oven at $65^{\circ} \mathrm{C}$ for $3 \mathrm{~h}$, then deparaffinized in $100 \%$ xylene and dehydrated with graded ethanol washes. Antigen retrieval was performed in a pressure cooker, followed by the treatment with $3 \%$ hydrogen peroxide for $15 \mathrm{~min}$ at room temperature to block endogenous peroxidase activity. Thereafter, the sections were incubated at $4^{\circ} \mathrm{C}$ overnight with anti-Bcl-2 (dilution, 1:20; cat.no. PAB7640; Abnova, Taipei, China). After being incubated at $37^{\circ} \mathrm{C}$ for $1 \mathrm{~h}$, these slides were washed three times in PBS and incubated with horseradish peroxidase-conjugated anti-rabbit antibody (dilution, 1:1,000; cat. no. PAB10822; Abnova) for $15 \mathrm{mins}$ at $37^{\circ} \mathrm{C}$. The stained specimens were exposed to the 3,3-diaminobenzidine and counterstained with hematoxylin for $20 \mathrm{~min}$ at room temperature. For the negative controls, primary antibodies were replaced with PBS.

Bcl-2 staining was microscopically examined (Olympus Corporation, Tokyo, Japan; inverted fluorescent microscope; magnification, x100) and scored by two independent pathologists who were blind to the clinical data pertaining to the patients. A semi-quantitatively scoring system (0-3) was used to evaluate the expression level of Bcl-2. The intensity of the staining was classified as negative, weak, moderate or strong. Staining intensity was scored as follows: 0 (negative), 1 (weakly positive), 2 (moderately positive), and 3 (strongly positive). The proportion of each level of staining cells were estimated A, B, C and D (between 0-100\%). The above two scores were multiplied, the final score as follows: $(0 \times \mathrm{A} \%)+$ $(1 \times \mathrm{B} \%)+(2 \times \mathrm{C} \%)+(3 \times \mathrm{D} \%)$.

Statistical analysis. Statistical analyses were performed using the R statistical language with the 'survival' package. Briefly, the Chi-square test was performed to analyze the association between Bcl-2 expression and clinicopathological features. In the univariate survival analyses, the difference in median overall survival (OS) time between groups of patients was analyzed using the log-rank test. The independent prognostic factors of OS were further identified by multivariate Cox proportional hazards regression models. The hazard ratios (HRs) and 95\% confidence intervals (CIs) of the prognostic factors were calculated. Kaplan-Meier survival curves were constructed for survival analyses and differences were tested using the log-rank test. Bcl-2 expression was categorized as high or low using the median score. $\mathrm{P}<0.05$ was considered to indicate a statistically significant difference. 
Table I. Details of the 16 LUSC datasets used.

\begin{tabular}{|c|c|c|c|c|}
\hline Dataset ID & Platform & LUSC number & Country & Reference (PMID) \\
\hline gse3141 & GPL570 & 53 & USA & 16273092 \\
\hline gse 4573 & GPL96 & 130 & USA & 16885343 \\
\hline gse5828 & GPL3877 & 59 & Australia & 17601969 \\
\hline gse11117 & GPL6650 & 14 & Switzerland & 19833826 \\
\hline gse12428 & GPL1708 & 34 & Netherlands & 19334046 \\
\hline gse12472 & GPL1708 & 35 & Netherlands & 20832896 \\
\hline gse14814 & GPL96 & 52 & Canada & 20823422 \\
\hline gse17710 & GPL9053 & 56 & USA & 20643781 \\
\hline gse19188 & GPL570 & 24 & Netherlands & 20421987 \\
\hline gse29013 & GPL570 & 25 & USA & 21742808 \\
\hline gse37745 & GPL570 & 66 & Sweden & 23032747 \\
\hline gse30219 & GPL570 & 61 & France & 23698379 \\
\hline gse41271 & GPL6884 & 80 & USA & 23449933 \\
\hline gse11969 & GPL7015 & 35 & Japan & 16549822 \\
\hline gse50081 & GPL570 & 43 & Canada & 24305008 \\
\hline TCGA & GPL96 & 134 & USA & 22960745 \\
\hline
\end{tabular}

LUSC, lung squamous cell carcinoma; PMID, PubMed Identifier; TCGA, The Cancer Genome Atlas.

\section{Results}

Clinical features of patients with LUSC. A TMA containing 72 LUSC cases was utilized to perform IHC staining. Overall, 10 female patients and 62 male patients, with an age range of 41-86 years (mean age, 67.4 years) were included in the current study. According to the 7th edition of the AJCC TNM staging system, 53 patients $(73.6 \%)$ were at early stages (38 stage I and 15 stage II) and 19 patients (26.4\%) were at advanced stages (18 stage III and 1 stage IV). The diameter of the tumor of 16 patients $(22.5 \%)$ was $<3 \mathrm{~cm}$, while that of the remaining 55 patients $(77.5 \%)$ was $\geq 3 \mathrm{~cm}$. There were 25 patients (34.7\%) with positive lymph node metastasis and 47 patients $(65.3 \%)$ exhibited negative lymph node metastasis. The primary clinicopathological characteristics of these patients are listed in Table II. Generally, the overall follow-up durations ranged between 3.9 and 84.3 months. Forty-five patients were alive at the end of the follow-up and the OS rate was $62.5 \%$ in the present study.

Meta-analysis of prognostic significance of Bcl-2 in patients with LUSC. To gain a comprehensive and robust insight into the prognostic significance of Bcl-2 in LUSC, the LUSC tumor gene expression profiles and survival data of 901 patients from 16 datasets were assembled, and integrated. The prognostic association between the expression level of Bcl-2 and OS were independently evaluated in 16 datasets using $\mathrm{z}$-scores.

To minimize the confounding influence of batch effects and other limitations derived from pooling raw data or merging expression data across multiple studies, weighted Z-tests were used to combine independent $\mathrm{Z}$-scores of $\mathrm{Bcl}-2$ into a 'meta-z-score'. In different cohorts, adverse and favorable prognostic significance was associated with Bcl-2 expression, with the final meta-Z-score being -2.43 for Bcl-2 (Fig. 1). This suggested that high $\mathrm{Bcl}-2$ expression level is associated with
Table II. Summary of patient characteristics $(n=72)$.

\begin{tabular}{lcc}
\hline $\begin{array}{l}\text { Clinicopathological } \\
\text { features }\end{array}$ & $\begin{array}{c}\text { No. of } \\
\text { patients }\end{array}$ & $\begin{array}{c}\text { Percentage of } \\
\text { patients }\end{array}$ \\
\hline $\begin{array}{l}\text { Sex } \\
\text { Male }\end{array}$ & 62 & 86.1 \\
Female & 10 & 13.9 \\
Age, years & & \\
$<60$ & 19 & 26.4 \\
$\geq 60$ & 53 & 73.6 \\
Tumor size, cm & & \\
$<3$ & 17 & 23.6 \\
$\geq 3$ & 55 & 76.4 \\
Smoking & & \\
$<20$ & 21 & 29.2 \\
$\geq 20$ & 51 & 70.8 \\
Stage & & \\
I & 38 & 52.8 \\
II & 15 & 20.8 \\
III & 18 & 25.0 \\
IV & 1 & \\
\hline & & \\
\hline
\end{tabular}

longer survival times, and $\mathrm{Bcl}-2$ may serve as a prognostic biomarker in predicting the OS rate of patients with LUSC.

Association between the IHC expression of Bcl-2 and clinicopathological features. The expression of $\mathrm{Bcl}-2$ protein was analyzed in 72 patients with LUSC using IHC (Fig. 2), and it was revealed that $\mathrm{Bcl}-2$ protein was primarily localized to 


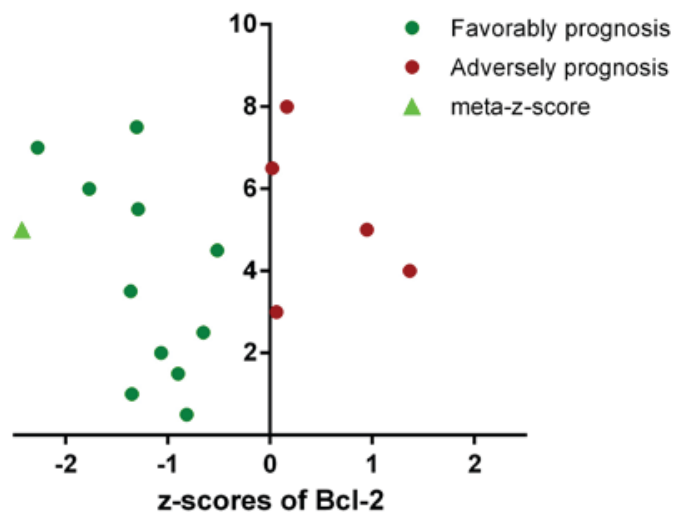

Figure 1. Z-score and meta-Z-score of Bcl-2. Meta-Z-score, -2.43. Bcl-2, B-cell lymphoma 2.

the cell membrane and cytoplasm of lung SCC cells, which is consistent with a previous study (18). In contrast, adjacent bronchial mucosa and alveolar epithelial cells were Bcl-2-negative. To minimize the bias of IHC scoring, a scoring standard was set up and two independent researchers scored all of IHC staining samples. Considering the overall positive rate of Bcl-2 expression observed in this study, patients with LUSC were divided into two groups as follows: Score $\leq 1$ as the low expression group and score $>1$ as the high expression group. The positive rate of $\mathrm{Bcl}-2$ expression in the current study was $91.7 \%(66 / 72)$, with $54.2 \%$ (39/72) of patients exhibiting weak expression (score $\leq 1$ ) and $45.8 \%$ (33/72) of patients exhibiting strong expression (score $>1$ ).

The association between $\mathrm{Bcl}-2$ protein expression and the clinicopathological parameters of patients with LUSC was analyzed using the Chi-square test (Table III). The results revealed that high $\mathrm{Bcl}-2$ expression was significantly associated with heavy smoking $(\mathrm{P}<0.05)$. No statistically significant difference was identified between Bcl-2 expression and other clinical parameters, including age, gender, tumor diameter, TNM stage or lymph node metastasis.

Survival analysis. All 72 patients were included in the survival analysis and the multivariate Cox proportional hazards model was applied to determine the effect of $\mathrm{Bcl}-2$ expression on survival. The Kaplan-Meier survival curves demonstrated that patients with LUSC with high Bcl-2 expression had a significantly favorable OS time (Fig. 3). Univariate survival analyses were employed to identify the difference between patients with LUSC with different Bcl-2 expression levels. The log-rank test revealed that Bcl-2 expression levels, clinical stages and tumor size were significantly associated with OS $(\mathrm{P}<0.05)$. In addition, multivariate analysis indicated that $\mathrm{Bcl}-2$ protein expression is an independent prognostic factor for patients with LUSC (HR, 0.295; CI, 0.097-0.904; P<0.05). Detailed data are listed in Table IV.

\section{Discussion}

The majority of patients with LUSC are diagnosed at advanced stages because there are no clinical symptoms or effective biomarkers (30). Additionally, numerous
Table III. Association between the immunohistochemical expression of $\mathrm{Bcl}-2$ and clinicopathological features.

\begin{tabular}{|c|c|c|c|c|}
\hline \multirow{2}{*}{$\begin{array}{l}\text { Clinicopathological } \\
\text { variables }(n=72)\end{array}$} & \multicolumn{2}{|c|}{$\mathrm{Bcl}-2$} & \multirow[b]{2}{*}{$\chi^{2}$} & \multirow[b]{2}{*}{ P-value } \\
\hline & Low & High & & \\
\hline \multicolumn{5}{|l|}{ Age, years } \\
\hline$<60$ & 10 & 9 & $2.40 \times 10^{-31}$ & $>0.99$ \\
\hline$\geq 60$ & 29 & 24 & & \\
\hline \multicolumn{5}{|l|}{ Sex } \\
\hline Female & 35 & 27 & 0.39 & 0.53 \\
\hline Male & 4 & 6 & & \\
\hline \multicolumn{5}{|l|}{ AJCC7 stage } \\
\hline I-II & 39 & 32 & 0.01 & 0.93 \\
\hline III-IV & 0 & 1 & & \\
\hline \multicolumn{5}{|l|}{ Smoking } \\
\hline$<20$ & 7 & 14 & 4.17 & 0.04 \\
\hline$\geq 20$ & 31 & 18 & & \\
\hline \multicolumn{5}{|l|}{ Tumor size, $\mathrm{cm}$} \\
\hline$<3$ & 11 & 5 & 4.75 & 0.19 \\
\hline $3-5$ & 13 & 15 & & \\
\hline $5-7$ & 8 & 10 & & \\
\hline$\geq 7$ & 7 & 2 & & \\
\hline \multicolumn{5}{|l|}{ Lymph node } \\
\hline NO & 24 & 23 & 1.45 & 0.48 \\
\hline \multicolumn{5}{|l|}{ Metastasis } \\
\hline $\mathrm{N} 1$ & 6 & 6 & & \\
\hline N2 & 9 & 4 & & \\
\hline
\end{tabular}

AJCC, American Joint Committee on Cancer; Bcl-2, B-cell lymphoma 2 .

patients with lung cancer are diagnosed at advanced stage; therefore, it is essential to seek highly sensitive and specific molecular markers of lung cancer for early diagnosis (31).

Bcl-2 as a prognostic marker of lung cancer, including small cell lung cancer, has been reported in numerous studies, but the results remain conflicted and controversial $(17,21)$. Furthermore, due to small sample sizes, detection methods inconsistencies and other limitations, it is difficult to compare the results (25). A meta-analysis published by Zhang et al (21) summarizes 50 articles that investigated the prognostic value of Bcl-2 in NSCLC, which, to the best of our knowledge, is currently the most comprehensive study. However, these datasets were divided into seven subtypes according to clinical or pathological stages and none of these studies were specific to LUSC.

To get a comprehensive analysis of Bcl-2 expression and prognosis, a robust survival meta-z approach was used to integrate multiple LUSC datasets from known databases (30). This method provided a larger sample size, reducing the potential for errors from single datasets. Additionally, the results were validated using IHC on a 72-sample TMA. The results suggested that Bcl-2 was significantly associated with the OS of patients 
Table IV. Cox proportional hazard regression model analysis.

\begin{tabular}{|c|c|c|c|c|c|c|}
\hline \multirow[b]{2}{*}{ Characteristic } & \multicolumn{3}{|c|}{ Univariate analysis } & \multicolumn{3}{|c|}{ Multivariate analysis } \\
\hline & $95 \% \mathrm{CI}$ & Log-rank & $\mathrm{P}>|\mathrm{z}|$ & $95 \% \mathrm{CI}$ & HR & $\mathrm{P}>|\mathrm{z}|$ \\
\hline Bcl-2 expression & $0.098-0.919$ & 19.790 & 0.001 & $0.097-0.904$ & 0.295 & 0.033 \\
\hline Age & $0.576-6.713$ & 1.210 & 0.281 & & & \\
\hline Gender & $0.555-5.016$ & 0.849 & 0.357 & & & \\
\hline Tumor size & $0.561-9.964$ & 11.632 & 0.009 & $0.719-2.217$ & 1.276 & 0.416 \\
\hline Stage & $0.822-9.827$ & 14.123 & 0.010 & $0.801-2.624$ & 1.142 & 0.219 \\
\hline
\end{tabular}

HR, hazard ratio; CI, confidence interval; Bcl-2, B-cell lymphoma 2.
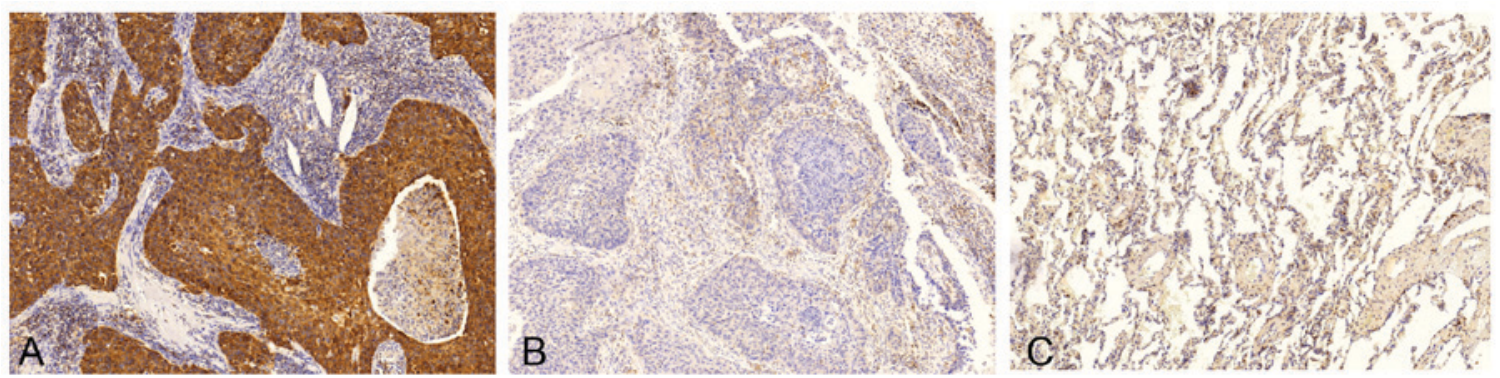

Figure 2. Expression of Bcl-2 in lung squamous cell carcinoma tissues as detected by IHC. Presentive figures of (A) high expression and (B) low expression of Bcl-2, and (C) negative control (magnification, x100). Bcl-2, B-cell lymphoma 2.

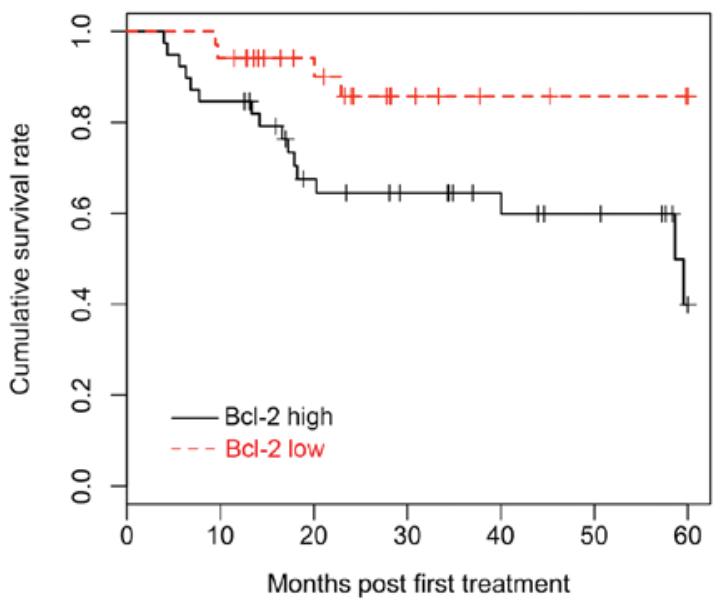

Figure 3. Kaplan-Meier overall survival analysis of patients with lung squamous cell carcinoma. Survival analysis was performed according to the expression status of Bcl-2 $(\mathrm{P}<0.05)$. Bcl-2, B-cell lymphoma 2.

with LUSC and may also serve as an independent prognostic factor as supported by multivariate analysis. Furthermore, Bcl-2 was associated with tumor size and TNM stage (Table IV), which are similar to the results observed in breast cancer (32).

Notably, there may be a dual role for Bcl-2 in cancer (33). Since Bcl-2 has anti-apoptotic effects based on in vitro and in vivo experiments, it is expected that high $\mathrm{Bcl}-2$ expression may lead to worse prognosis, rather than prolonged survival (11). However, high expression of $\mathrm{Bcl}-2$ was demonstrated to be a favorable prognosis factor in LUSC in the present study, suggesting that Bcl-2 may be involved in a feedback loop for cell regulation, and the exact role of $\mathrm{Bcl}-2$ in the regulation of apoptosis may depend on the cell environment (34).

In conclusion, the results of the present study suggest that high Bcl-2 expression in patients with LUSC indicates favorable prognosis, indicating $\mathrm{Bcl}-2$ could be a potential prognostic biomarker for LUSC.

\section{Acknowledgements}

Not applicable.

\section{Funding}

This study is founded by the National High Technology Research and Development Program of China (863 Program) (grant no. ss2014AA020602 and ss2014AA020604).

\section{Availability of data and materials}

All data generated or analyzed during this study are included in this published article.

\section{Authors' contributions}

CF, FY, XY, MQ and JunW designed the study; JiaW and SH performed the bioinformatics analysis. SG and JinW conducted the TAM staining experiments. CF, XY and MQ wrote and revised the manuscript. 


\section{Ethics approval and consent to participate}

The present study was approved by the Institutional Review Board of the People's Hospital of Peking University and written informed consent was obtained from each patient.

\section{Consent for publication}

Written informed consent was obtained from all patients for the publication of their data.

\section{Competing interests}

The authors declare that they have no competing interests.

\section{References}

1. Siegel RL, Miller KD and Jemal A: Cancer statistics, 2016. CA Cancer J Clin 66: 7-30, 2016

2. Ferlay J, Soerjomataram I, Dikshit R, Eser S, Mathers C, Rebelo M, Parkin DM, Forman D and Bray F: Cancer incidence and mortality worldwide: Sources, methods and major patterns in GLOBOCAN 2012. Int J Cancer 136: E359-E386, 2015.

3. Lewis DR, Check DP, Caporaso NE, Travis WD and Devesa SS: US lung cancer trends by histologic type. Cancer 120: 2883-2892, 2014.

4. Bild AH, Yao G, Chang JT, Wang Q, Potti A, Chasse D, Joshi MB, Harpole D, Lancaster JM, Berchuck A, et al: Oncogenic pathway signatures in human cancers as a guide to targeted therapies. Nature 439: 353-357, 2006.

5. Huang P, Cheng CL, Chang YH, Liu CH, Hsu YC, Chen JS, Chang GC, Ho BC, Su KY, Chen HY and Yu SL: Molecular gene signature and prognosis of non-small cell lung cancer. Oncotarget 7: 51898-51907, 2016.

6. Tan Q, Wang G, Huang J, Ding Z, Luo Q, Mok T, Tao Q and Lu S Epigenomic analysis of lung adenocarcinoma reveals novel DNA methylation patterns associated with smoking. Onco Targets Ther 6: 1471-1479, 2013.

7. Botling J, Edlund K, Lohr M, Hellwig B, Holmberg L, Lambe M, Berglund A, Ekman S, Bergqvist M, Pontén F, et al: Biomarker discovery in non-small cell lung cancer: Integrating gene expression profiling, meta-analysis, and tissue microarray validation Clin Cancer Res 19: 194-204, 2013.

8. Wilkerson MD, Yin X, Hoadley KA, Liu Y, Hayward MC, Cabanski CR, Muldrew K, Miller CR, Randell SH, Socinski MA, et al: Lung squamous cell carcinoma mRNA expression subtypes are reproducible, clinically important, and correspond to normal cell types. Clin Cancer Res 16: 4864-4875, 2010.

9. Raponi M, Zhang Y, Yu J, Chen G, Lee G, Taylor JM, Macdonald J, Thomas D, Moskaluk C, Wang Y and Beer DG: Gene expression signatures for predicting prognosis of squamous cell and adenocarcinomas of the lung. Cancer Res 66: 7466-7472, 2006.

10. Lawson MH, Cummings NM, Rassl DM, Vowler SL, Wickens M Howat WJ, Brenton JD, Murphy G and Rintoul RC: Bcl-2 and $\beta 1$-integrin predict survival in a tissue microarray of small cell lung cancer. Br J Cancer 103: 1710-1715, 2010.

11. Dutta C, Day T, Kopp N, van Bodegom D, Davids MS, Ryan J, Bird L, Kommajosyula N, Weigert O, Yoda A, et al: BCL-2 suppresses PARP1 function and nonapoptotic cell death. Cancer Res 72: 4193-4203, 2012.

12. Janumyan Y, Cui Q, Yan L, Sansam CG, Valentin M and Yang E: G0 function of BCL2 and BCL-xL requires BAX, BAK, and p27 phosphorylation by Mirk, revealing a novel role of BAX and BAK in quiescence regulation. J Biol Chem 283: 34108-34120, 2008.

13. Daniel JC and Smythe WR: The role of Bcl-2 family members in non-small cell lung cancer. Semin Thorac Cardiovasc Surg 16: 19-27, 2004.

14. Bouchalova K, Kharaishvili G, Bouchal J, Vrbkova J, Megova M and Hlobilkova A: Triple negative breast cancer-BCL2 in prognosis and prediction. Review. Curr Drug Targets 15: 1166-1175, 2014.
15. Renouf DJ, Wood-Baker R, Ionescu DN, Leung S, Masoudi H, Gilks CB and Laskin J: BCL-2 expression is prognostic for improved survival in non-small cell lung cancer. $\mathrm{J}$ Thorac Oncol 4: 486-491, 2009

16. Martinez-Arribas F, Alvarez T, Del Val G, Martín-Garabato E, Núñez-Villar MJ,Lucas R, Sánchez J, Tejerina A and Schneider J: Bcl-2 expression in breast cancer: A comparative study at the mRNA and protein level. Anticancer Res 27: 219-222, 2007.

17. Martin B, Paesmans M, Berghmans T, Branle F, Ghisdal L, Mascaux C, Meert AP, Steels E, Vallot F, Verdebout JM, et al: Role of Bcl-2 as a prognostic factor for survival in lung cancer: A systematic review of the literature with meta-analysis. Br J Cancer 89: 55-64, 2003

18. Shibata Y, Hidaka S, Tagawa $\mathrm{Y}$ and Nagayasu T: Bcl-2 protein expression correlates with better prognosis in patients with advanced non-small cell lung cancer. Anticancer Res 24: 1925-1928, 2004.

19. Porebska I, Kosacka M, Wyrodek E and Jankowska R: Expression of p53, bcl-2 and nm23 proteins in squamous cell lung cancer. Pneumonol Alergol Pol 77: 131-137, 2009 (In Polish).

20. Kim YC, Park KO, Kern JA, Park CS, Lim SC, Jang AS and Yang JB: The interactive effect of Ras, HER2, P53 and Bcl-2 expression in predicting the survival of non-small cell lung cancer patients. Lung Cancer 22: 181-190, 1998.

21. Zhang J, Wang S, Wang L, Wang R, Chen S, Pan B, Sun Y and Chen H: Prognostic value of Bcl-2 expression in patients with non-small-cell lung cancer: A meta-analysis and systemic review. Onco Targets Ther 8: 3361-3369, 2015.

22. Cancer Genome Atlas Research Network: Comprehensive genomic characterization of squamous cell lung cancers. Nature 489: 519-525, 2012.

23. Cancer Genome Atlas Research Network: Comprehensive molecular profiling of lung adenocarcinoma. Nature 511: 543-550, 2014.

24. Baty F, Facompré M, Kaiser S, Schumacher M, Pless M, Bubendorf L, Savic S, Marrer E, Budach W, Buess M, et al: Gene profiling of clinical routine biopsies and prediction of survival in non-small cell lung cancer. Am J Respir Crit Care Med 181: 181-188, 2010.

25. Wang Y, Zhao W and Zhou X: Integration of genomic data analysis for demonstrating potential targets in the subgroup populations of squamous cell lung cancer patients. Oncotarget, Jun 15, 2016. DOI: 10.18632/oncotarget.10072.

26. Gentles AJ, Newman AM, Liu CL, Bratman SV, Feng W, Kim D, Nair VS, Xu Y, Khuong A, Hoang CD, et al: The prognostic landscape of genes and infiltrating immune cells across human cancers. Nat Med 21: 938-945, 2015.

27. Pøhl M, Olsen KE, Holst R, Ditzel HJ and Hansen O: Tissue microarrays in non-small-cell lung cancer: Reliability of immunohistochemically-determined biomarkers. Clin Lung Cancer 15: 222-230.e3, 2014.

28. Edge SB and Compton CC: The American joint committee on cancer: The 7th edition of the AJCC cancer staging manual and the future of TNM. Ann Surg Oncol 17: 1471-1474, 2010.

29. Micke P, Mattsson JS, Djureinovic D, Nodin B, Jirström K, Tran L, Jönsson P, Planck M, Botling J and Brunnström H: The impact of the 4th edition of the WHO classification of lung tumours on histological classification of resected pulmonary NSCCs. J Thorac Oncol 11: 862-872, 2016.

30. Li Q, Hou J, Hu Z, Gu B and Shi Y: Multiple mutations of lung squamous cell carcinoma shared common mechanisms. Oncotarget 7: 79629-79636, 2016.

31. Der SD, Sykes J, Pintilie M, Zhu CQ, Strumpf D, Liu N, Jurisica I, Shepherd FA and Tsao MS: Validation of a histology-independent prognostic gene signature for early-stage, non-small-cell lung cancer including stage IA patients. J Thorac Oncol 9: 59-64, 2014.

32. Hwang KT, Woo JW, Shin HC, Kim HS, Ahn SK, Moon HG, Han W, Park IA and Noh DY: Prognostic influence of BCL-2 expression in breast cancer. Int J Cancer 131: E1109-E1119, 2012.

33. Peng Y, Wang L, Qing Y, Li C, Ren T, Li Q, Li M, Zhang S, Shan J, Wang G, et al: Polymorphisms of BCL2 and BAX genes associate with outcomes in advanced non-small cell lung cancer patients treated with platinum-based chemotherapy. Sci Rep 5: 17766, 2015.

34. Singh K and Briggs JM: Functional implications of the spectrum of BCL2 mutations in lymphoma. Mutat Res Rev Mutat Res 769: 1-18, 2016.

This work is licensed under a Creative Commons Attribution-NonCommercial-NoDerivatives 4.0 International (CC BY-NC-ND 4.0) License. 